\title{
Increased expression of IREI $\alpha$ and stress-related signal transduction proteins in ischemia-reperfusion injured retina
}

\author{
Natsuyo Hata' \\ Toshiyuki Oshitari ${ }^{1,2}$ \\ Akiko Yokoyama ${ }^{1,3}$ \\ Yoshinori Mitamura' \\ Shuichi Yamamoto' \\ 'Department of Ophthalmology \\ and Visual Science, Chiba University \\ Graduate School of Medicine, \\ Chuo-ku, Chiba, Japan; ${ }^{2}$ Department \\ of Ophthalmology, Kimitsu Central \\ Hospital, Kisarazu City, Chiba, Japan; \\ ${ }^{3}$ Department of Ophthalmology, \\ Inoue Memorial Hospital, Chuo-ku, \\ Chiba, Japan
}

\begin{abstract}
The purpose of this study was to determine whether the expression of ER stress-related factors IRE1 $\alpha$, apoptosis signal-regulating kinase 1 (ASK1), SAPK/ERK kinase 1 (SEK1) and c-Jun N-terminal kinase ( $\mathrm{NNK}$ ) is associated with the damaged retinal neurons induced by ischemia-reperfusion injury. After 60 minutes of ischemia, the rat retinas were reperfused, and retinas were isolated and fixed after 6, 9, 12, 18, and 24 hours, and 2, 5, and 9 days of reperfusion. Cryosections were immunostained with Fluoro-Jade B, a degenerating neuron marker to label degenerating neurons. Semi-quantitative analysis of the expression of IRE1 $\alpha$, ASK1, SEK1, and JNK were performed in both control and ischemic retinas. In ischemic retinas, the intensities of IRE1 $\alpha$ immunoreactivity in the ganglion cell layer (GCL) were significantly higher than in the control retinas. In ischemic retinas, the numbers of SEK1-, ASK1-, and JNK-positive cells were significantly increased in the GCL compared to those in the control retinas. In addition, the cells that were positive for SEK1-, ASK1-, and JNK were also positive for Fluoro-Jade B-positive cells. These results indicate that the increased expression of ER stress-related factors was, in part, associated with the retinal neuronal abnormalities after ischemia-reperfusion injury in rat retinas.
\end{abstract}

Keywords: endoplasmic reticulum, IRE1 $\alpha$, apoptosis signal-regulating kinase 1, SAPK/ERK kinase 1, c-Jun N-terminal kinase, Fluoro-Jade B, ischemia-reperfusion injury

\section{Introduction}

The endoplasmic reticulum (ER) is an intracellular organelle that is associated with the synthesis and folding of proteins and the post-translational modification of proteins (Rao et al 2004; Lindholm et al 2006). The accumulation of unfolded proteins or upset in the $\mathrm{Ca}^{2+}$ homeostasis in the ER can trigger ER stress-induced apoptosis (Rao et al 2004; Lindholm et al 2006). Recent studies have shown that the ER stress-induced apoptosis is associated with the pathogenesis of various human diseases, eg, Parkinson's disease (Imai and Takahashi 2004), amyotrophic lateral sclerosis (Tobisawa et al 2003; Wootz et al 2004), polyglutamine diseases (Nishitoh et al 2002), diabetes mellitus (Oshitari et al 2008a), and acute brain disorders (Larner et al 2004; Tajiri et al 2004; Hayashi et al 2005).

Apoptosis of retinal neurons is a progressive, irreversible process that occurs following ischemic injury to the retina, eg, central or branch retinal artery occlusion (Zhang et al 2005) and ischemic optic neuropathy (Levin and Louhab 1996). To rescue the retinal neurons after ischemia and to establish neuroprotective therapies for ischemic diseases, information on the precise mechanism causing the neuronal apoptosis is required. However, the precise mechanism for retinal neuronal apoptosis has not been determined yet.

The results of recent studies showed that one of the ER stress-mediated pathways, the $\mathrm{C} / \mathrm{EBP}$ homologous protein ( $\mathrm{CHOP})$-mediated pathway, is involved in the death 
of retinal neurons following $N$-methyl-D-aspartate (NMDA) treatment (Awai et al 2006, Shimazawa et al 2007). The results of Awai and colleagues' study showed that CHOPdeficient mice were more resistant to the NMDA-induced retinal injury (Awai et al 2006). Another study showed that the retinal ganglion cells (RGCs) of apoptosis signalregulating kinase 1 (ASK1)-knockout mice (Harada et al 2006) were less susceptible to ischemia-reperfusion injury. These findings suggested that ASK1 is associated with the $\mathrm{RGC}$ death in ischemia-reperfusion retinas.

The ASK1-c-Jun N-terminal kinase (JNK) pathway is known to be one of the major ER stress-induced apoptotic pathways in the central nervous system (Nishitoh et al 2002). However, the earlier study in the retina did not focus on the relationship between the ER stress and the ASK1-mediated pathway but on the ASK1-p38 pathway (Harada et al 2006).

We have reported that the electrical translocation of the heat shock protein 27 (Hsp 27) into RGCs reduced the damage of RGCs after ischemia-reperfusion injury in rat retinas (Yokoyama et al 2001). It is known that chaperon Hsp 27 stabilizes the cytoskeleton and limits the accumulation of unfolded proteins by inhibiting protein synthesis. This is relevant because the accumulation of unfolded proteins in the ER can induce ER stress-mediated apoptosis, and an increase of intracellular $\mathrm{Ca}^{2+}$ can activate SAPK/ERK kinase 1 (SEK1) also called the MAP kinase/extracellular signal-regulated kinase 4 (MEK4)-mediated cell death pathway (Dolmetsch et al 1997).

The purpose of this study was to determine whether the increased expression of ER stress-related factor IRE1 $\alpha$, ASK1, SEK1/MEK4, and JNK is associated with damaged neurons after ischemia-reperfusion injury in rat retinas.

\section{Methods}

\section{Animals}

The experiments were conducted on adult male Wistar (200-250 g) rats, which were housed three/cage in a temperature-controlled room and had free access to food and water. The procedures used on the animals were in accordance with the ARVO Statement for the Use of Animals in Ophthalmic and Vision Research. The experimental protocols were approved by the Animal Use Committee of Chiba University Graduate School of Medicine.

\section{Induction of Ischemia}

The ischemia-reperfusion in the rats was done as reported in detail previously (Yokoyama et al 2001). Briefly, under deep anesthesia performed by an intraperitoneal injection of a mixture of $500 \mathrm{mg}$ urethane/kilogram body weight, $11 \mathrm{mg} / \mathrm{kg}$ ketamine, and $14 \mathrm{mg} / \mathrm{kg}$ xylazine, the left optic nerve was exposed through an incision in the upper conjunctival fornix and detachment of the superior rectus muscle. The eyeball was retracted, and the dural sheath surrounding the optic nerve was carefully cut with microscissors to separate the central retinal artery from the nerve. The central retinal artery with the surrounding tissue was clamped with a cerebral aneurysm surgical clip. After 60 minutes, the clip was removed to allow reperfusion of the retinal vessels. The absolute cessation and reperfusion of blood flow in the retinal vessels was confirmed by ophthalmoscopy. Only those eyes, in which reperfusion was confirmed within 5 minutes after the clip was removed, were used. During these procedures, the body temperature of the rats was maintained at approximately $37^{\circ} \mathrm{C}$.

\section{Histological processing}

After 6, 9, 12, 18, and 24 hours, and 2, 5, and 9 days of reperfusion, the animals were deeply anesthetized and killed by an intracardiac perfusion of $4 \%$ paraformaldehyde in $0.1 \mathrm{M}$ phosphate-buffered saline (PBS). The retinas were removed from the eyecup of both eyes, postfixed in the same fixative for 3 hours, and embedded in optimal cutting temperature compound (Tissue Tek; Miles, Elkhart, IN). The control right eyes and ischemia-reperfusion left eyes were cut on a freezing sliding microtome at a thickness of $12 \mu \mathrm{m}$.

\section{Immunohistochemistry}

The sections were rinsed with PBS, then stained with rabbit anti-phospho-JNK (p-JNK) (1:50; Santa Cruz Biotechnology, Santa Cruz, CA; cat\# sc-12882), or anti-ASK1 (1:100; Santa Cruz Biotechnology; cat\# sc-7931), or anti-phospho-SEK1/ MEK4 (p-SEK1/MEK4) (1:400; Santa Cruz Biotechnology; cat\# sc-7990), or anti-IRE1 $\alpha$ (1:50; Santa Cruz Biotechnology; cat\# sc-20790) as the primary antibody, and Texas-Redconjugated goat antibody raised against rabbit $\operatorname{IgG}(1: 1000$; Antibodies Incorporated, Davis, CA) as the secondary antibody. For controls, the primary antibodies were omitted in the staining. All sections were stained with Fluoro-Jade B (Chemicon International, Temecula, CA) to label degenerating neurons and with 4,6-diamidino-2-phenyl-indole (DAPI) to label the nuclei. The sections were examined and photographed with a fluorescent microscope (Carl Zeiss, Jena, Germany).

\section{Semi-quantitative analysis of IREI $\alpha$ immunoreactivity in the ganglion cell layer}

Texas Red intensities of IRE1 $\alpha$ in the ganglion cell layer (GCL) were examined by NIH image analysis software. Six animals/ 
group were examined for the semi-quantitative analysis. One hundred fields from 20 sections/animal were examined for the quantitative analysis for IRE1 $\alpha$ expression in the retinas. The analyses were done by the same two observers who were masked to the experimental procedures performed on the rats. Data are expressed as the means \pm standard deviations (SDs). Statistical analyses were performed with the Mann-Whitney $\mathrm{U}$ test. A $P<0.05$ was considered to be significant.

\section{Semi-quantitative analysis of expression of ASK I, p-SEKI/MEK4, and p-JNK in the GCL}

The numbers of ASK1-, p-SEK1/MEK4-, p-JNK-, and Fluoro-Jade B-positive cells in the GCL were counted/unit area in all groups. In addition, the numbers of ASK1-, p-SEK1/MEK4-, and p-JNK-positive cells that were double stained with Fluoro-Jade B were counted in the GCL. Six animals/group were examined for the semi-quantitative analysis. Fluoro-Jade B-positive cells were also counted, and the time course in the number of Fluoro-Jade B-positive cells after reperfusion was determined. One hundred fields were counted for each group of rats. The counting and analyses were done by the same two masked observers. The data are presented as the means \pm SDs. Statistical analysis was performed by the Mann-Whitney $\mathrm{U}$ test. A $P<0.05$ was considered to be significant.

\section{Western blot analysis}

Western blot analysis was performed for the quantitative analysis of IRE1 $\alpha$ expression in ischemic retinas. Briefly, retinas were isolated and incubated in $25 \mathrm{mM}$ Tris, pH7.4, containing $1 \mathrm{mM}$ EDTA, $1 \mathrm{mM}$ phenylmethylsulfonyl fluoride and $0.1 \%$ Triton X-100. Western blots were performed with $25 \mu \mathrm{g}$ protein per lane and transferred onto nitrocellulose membrane according to Towbin and colleagues' procedure (Towbin et al 1979). After blocking with 5\% fat-free milk in tribromosalicylanilide, the membrane was incubated with the blocking solution containing rabbit anti-IRE $1 \alpha$ antibody (1:500) overnight. After washing, the membrane was incubated with anti-rabbit antibody conjugated with horseradish peroxidase $(1: 15000)$ for 1 hour. The Immuno-Star Chemiluminescent Protein Detection System was used to detect the protein level of IRE1 $\alpha$. To determine the $\beta$-actin signals, the membrane that had been exposed to IRE1 $\alpha$ antibody was stripped with a buffer containing $62.5 \mathrm{mM}$ Tris, $\mathrm{pH} 6.8,2 \%$ SDS, and $100 \mathrm{mM}$ 2-Mercaptoethanol. After washing and blocking, the membrane was incubated with $\beta$-actin antibody solution (1:1000) overnight. After washing, the membrane was incubated with antimouse antibody conjugated with horseradish peroxidase (1:10000) for 1 hour, applied to the Immuno-Star Chemiluminescent substrate and exposed to X-ray film. Densitometric analysis of the luminescent signal was performed at nonsaturating exposures with a scanning densitometer and NIH image software. The data are presented as the means \pm SDs. Statistical analysis was performed by the Mann-Whitney $\mathrm{U}$ test. A $P<0.05$ was considered to be significant.

\section{Results}

\section{Time course of Fluoro-Jade B positive cells in the GCL}

To examine the time course of the degenerating neurons in the ischemic retinas, we counted the number of Fluoro-Jade B-positive cells in the GCL after different reperfusion times (Figure 1). Fluoro-Jade B-positive cells were first detected at 6 hours after reperfusion, and the number peaked 2 days after reperfusion in the GCL (Figure 1).

\section{Intensities of IREI $\alpha$ expression in ischemia-injured retinas}

To determine whether the altered IRE1 $\alpha$ expression was related to the pathogenesis of retinal ischemia, the retinal sections were immunostained for IRE1 $\alpha$. In control retinas, the expression of IRE1 $\alpha$ was detected in the GCL (Figure 2). In the ischemic retinas, the intensities of IRE $1 \alpha$ expression in the GCL were significantly increased compared to that in the control retinas $\left(179.8 \pm 16.3\right.$ pixels $/ \mathrm{mm}^{2}$ versus $147.6 \pm 26.9$ pixels $/ \mathrm{mm}^{2}, P=0.0011$; Figures 2 and 3$)$.

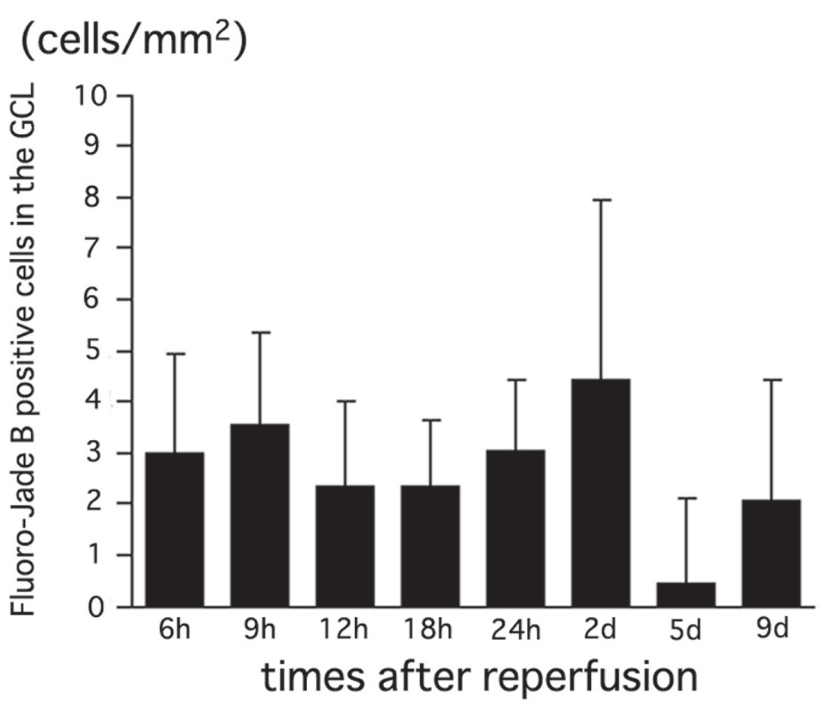

Figure I Time course of the number of Fluoro-jade B-positive cells in the GCL.The number of Fluoro-Jade B positive cells in the GCL peaks at 2 days after reperfusion. Abbreviations: h, hours; d, days. 


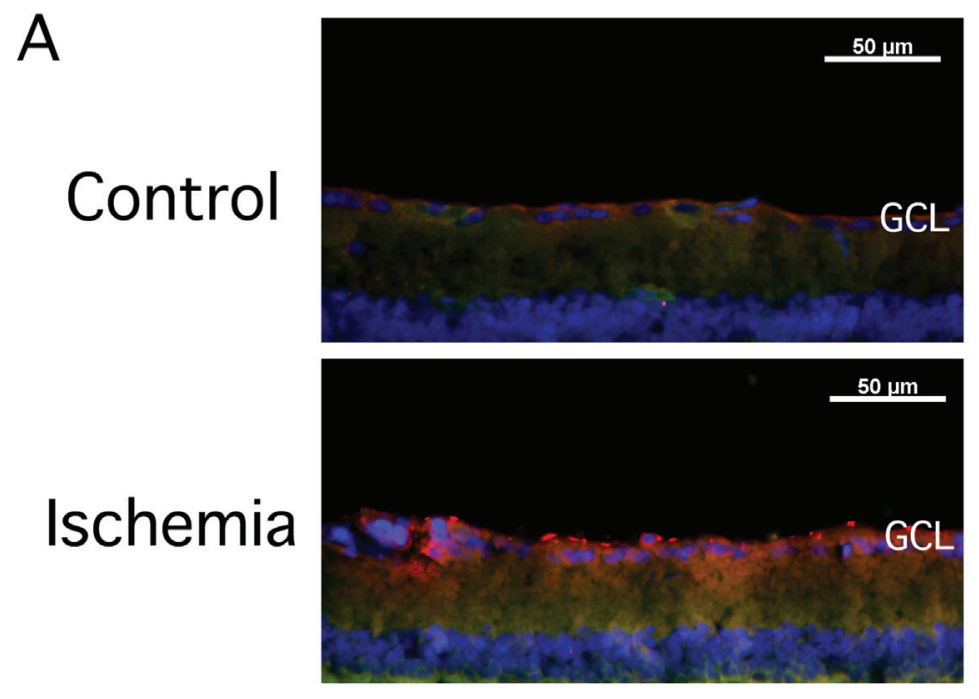

B

\section{Ischemia}
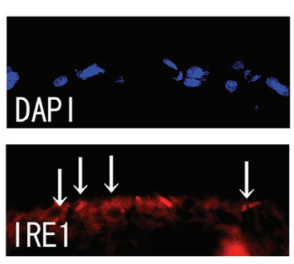
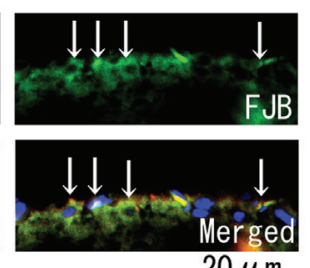

Control
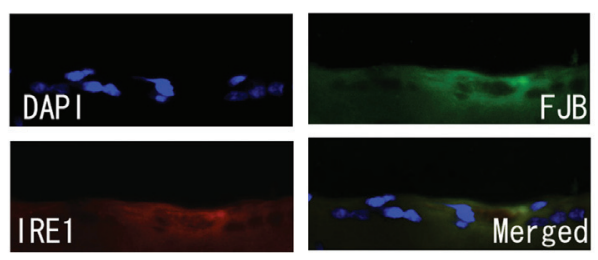

Figure 2 Expression of IREI $\alpha$ in control and ischemic retinas. The section was obtained from the ischemic retina 6 hours after reperfusion. In the ischemic retina, the expression of IREI $\alpha$ in the GCL is significantly stronger than in the control retinas (A) (merged). Although part of the IREI $\alpha$ expression was detected with Fluoro-Jade B positive signals (B) (multi panel), most IREI $\alpha$ expression did not co-exist with Fluoro-Jade B positive signals (A).

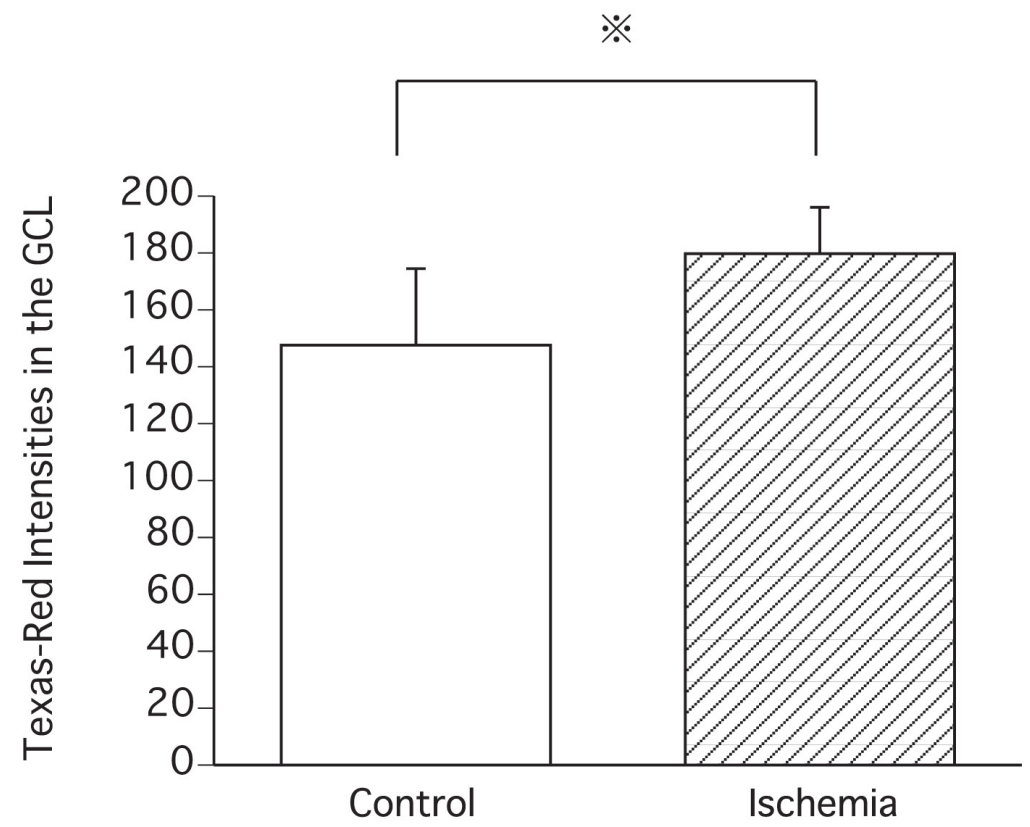

Figure 3 Intensities of IREI $\alpha$ expression in control and ischemic retinas. Texas-Red intensities of IREI $\alpha$ expression in the GCL of ischemic retinas are significantly increased compared to that of the control retinas ( $179.8 \pm 16.3$ pixels $/ \mathrm{mm}^{2}$ versus $147.6 \pm 26.9$ pixels $/ \mathrm{mm}^{2}, P=0.00 I I$ by Mann Whitney $U$ test). 
The increased expression of IRE $1 \alpha$ was present for 9 days after reperfusion. Most of the IRE1 $\alpha$ signals were independent of Fluoro-Jade B positive signals.

\section{IREI $\alpha$ protein levels}

\section{in ischemia-reperfusion-injured retinas}

To examine the levels of the IRE1 $\alpha$ protein in ischemic retinas, western blot analysis for IRE $1 \alpha$ was performed. In ischemic retinas, the IRE1 $\alpha$ protein level was significantly increased compared to that of the control retinas (159.9 \pm $73.9 \%$ of control versus $100 \pm 15.9 \%$ of control, $P=0.0369$; Figure 4). All densitometric values for western blot analysis were normalized to the $\beta$-actin signals (Figure 4 ). These results were consistent with the results of immunohistochemistry of IRE $1 \alpha$ expression in the retinas.

\section{Expression of ASKI in ischemic retinas}

In the control retinas, few cells expressed ASK1, but in the ischemia-reperfusion retinas, ASK1 was expressed

\section{Control Ischemia}

\author{
IRE1 $\alpha$
}

$\beta$-actin
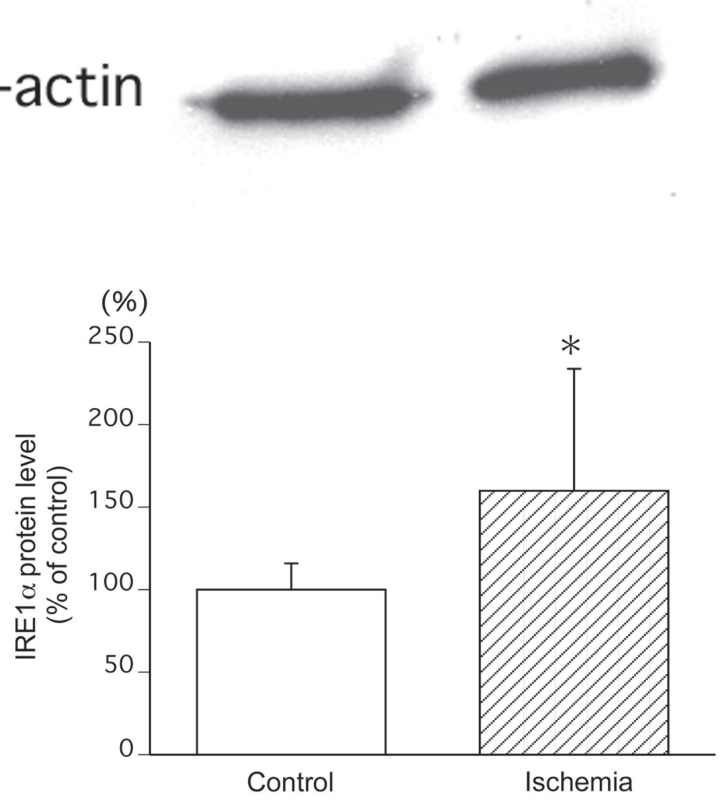

Figure 4 Western blot analysis of IREI $\alpha$ protein levels in ischemic retinas. In the ischemic retinas, the IREI $\alpha$ protein level was significantly increased compared to that of the control retinas $(100 \pm 15.9 \%$ of control versus $159.9 \pm 73.9 \%$ of control, $P=0.0369$ by Mann-Whitney $U$ test). All densitometric values for western blot analysis were normalized with $\beta$-actin signals. in the cells in the GCL (Figure 5A-C). The number of ASK1-positive cells in the GCL in the ischemic retinas after 6 hours of reperfusion was significantly larger than that in the control retinas $\left(1.74 \pm 1.24\right.$ cells $/ \mathrm{mm}^{2}$ versus $0.11 \pm 0.33$ cells $/ \mathrm{mm}^{2}, P=0.0007$ by Mann Whitney U test; Figure 5A). In addition, the number of ASK1 positive cells that co-localized with Fluoro-Jade B positive cells in the ischemic retinas after 6 hours of reperfusion was significantly larger than that of the control retinas $\left(0.9 \pm 1.0\right.$ cells $/ \mathrm{mm}^{2}$ versus $0.0 \pm 0.0$ cells $/ \mathrm{mm}^{2}, P=0.0307$ by Mann Whitney $\mathrm{U}$ test; Figure 5A). After 9 hours of reperfusion, there was no statistical significant difference in the number of ASK1 positive cells and ASK1/Fluoro-Jade B positive cells between the ischemic and control retinas. In ischemic retinas, more than one-half of the cells that were ASK1-positive were also Fluoro-Jade B-positive in the GCL (Figure 5B). The FluoroJade $\mathrm{B}$ positive cells had large soma which is a characteristic of RGCs (Figure 5C).

\section{P-SEK I/MEK4 expression in ischemic retinas}

In control retinas, few cells expressed p-SEK1/MEK4, but in the ischemic retinas, $\mathrm{p}-\mathrm{SEK} 1 / \mathrm{MEK} 4$ was expressed in the cells in the GCL (Figure 6A-C). The numbers of p-SEK1/MEK4 positive cells in the GCL in the ischemic retinas after 6 and 9 hours of reperfusion were significantly increased compared to that in the control retinas $(2.05 \pm$ 1.1 cells $/ \mathrm{mm}^{2}$ versus $0.27 \pm 0.47$ cells $/ \mathrm{mm}^{2}, P<0.0001$; $2.18 \pm 0.83$ versus $0.18 \pm 0.41$ cells $/ \mathrm{mm}^{2}, P=0.0162$ by Mann Whitney $U$ test, respectively; Figure 6A). In the ischemic retinas, the number of $\mathrm{p}-\mathrm{SEK} 1 / \mathrm{MEK} 4$ positive cells that co-localized with Fluoro-Jade B positive signals was significantly increased compared with that of the control retinas only after 6 hours of reperfusion $(1.24 \pm$ 0.83 cells $/ \mathrm{mm}^{2}$ versus $0.18 \pm 0.4$ cells $/ \mathrm{mm}^{2}, P=0.0004$ by Mann Whitney U test; Figure 6A). More than one-half of the cells expressing p-SEK1/MEK4 were also Fluoro-Jade B-positive (Figure 6B). The Fluoro-Jade B positive cells had large soma (Figure 6C).

\section{$\mathrm{P}-\mathrm{JNK}$ expression in ischemic retinas}

In control retinas, few p-JNK-positive cells were detected. In ischemic retinas, however, many $\mathrm{p}$-JNK positive cells were detected in the GCL and the inner plexiform layer and only a few in the inner nuclear layer (INL) and the outer plexiform layer (OPL; Figure 7A-E). The number of p-JNK positive cells in the GCL in the ischemic retinas only after 6 hours of reperfusion was significantly increased compared to that 
A

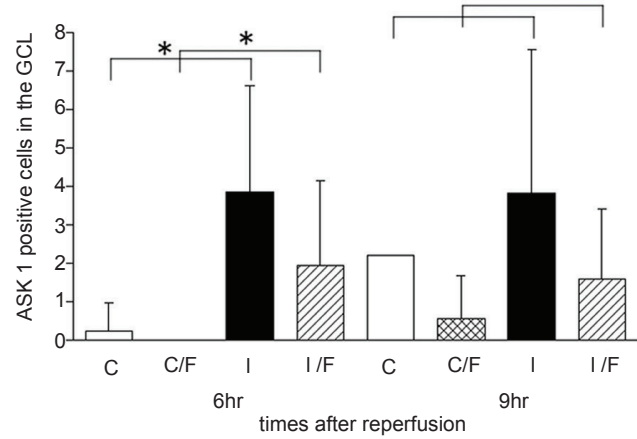

C Ischemia
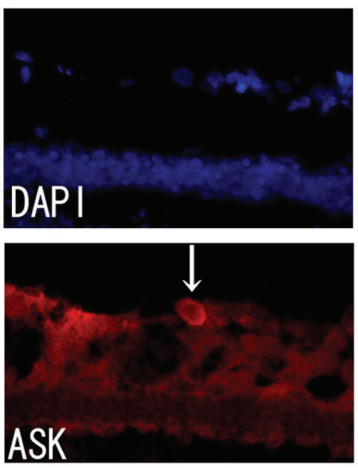
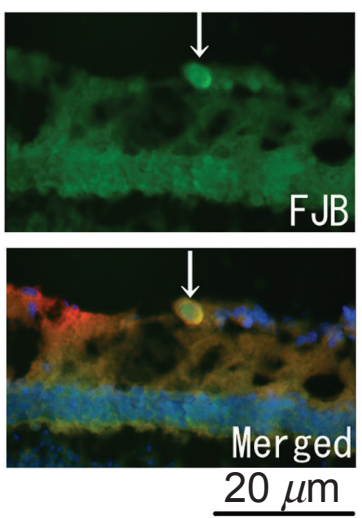

B

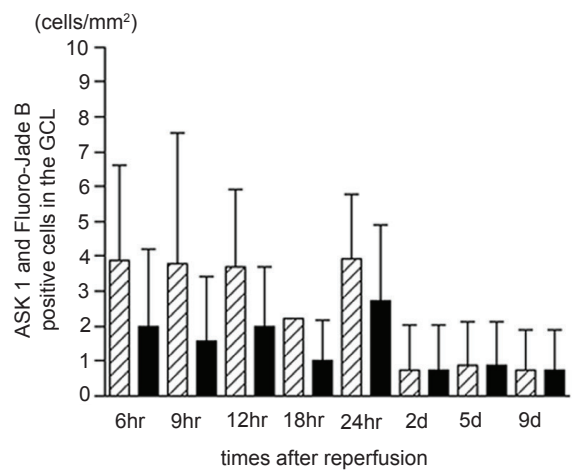

Control
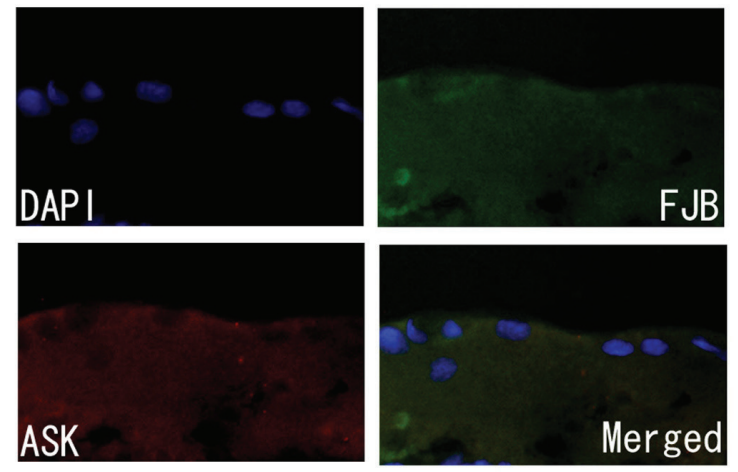

Figure 5 ASKI expression co-localized with Fluoro-Jade B-positive cells in ischemic retinas and the time course of the number of ASKI-positive cells that are also Fluoro-Jade B-positive. The number of ASKI-positive cells and the number of ASKI positive cells co-colocalized with Fluoro-Jade B positive signals in the ischemic retinas were significantly increased compared to that of the control retinas only after 6 hours of reperfusion (A). In ischemic retinas, more than one-half of the cells that were ASKI-positive were also Fluoro-Jade B-positive in the GCL (B). The sections were obtained from an ischemic retina 6 hour after reperfusion (C) (multi panel). The expression of ASKI co-existed with Fluoro-Jade B-positive cells in the GCL (arrows) (C).(A);White bars showed the number of ASKI positive cells in the control retinas and checked bars showed the number of ASK I positive and Fluoro-Jade B positive cells in the control retinas. Black bars showed the number of ASKI positive cells in the ischemic retinas and stripe bars showed ASK I positive and Fluoro-Jade B positive cells in the ischemic retinas. C, control; I, Ischemia; C/F, Control (ASKI) co-existed with Fluoro-Jade B; I/F, Ischemia (ASKI) co-existed with Fluoro-Jade B. Notes: $* P<0.05$ by Mann Whitney $U$ test. (B); Stripe bars showed the number of ASKI-positive cells and black bars showed the number of ASKI-positive cells co-existed with Fluoro-Jade B positive cells in the GCL of ischemic retinas.

Abbvreviations: h, hours; d, days.

of the control retinas $\left(11.7 \pm 8.4\right.$ cells $/ \mathrm{mm}^{2}$ versus $1.86 \pm$ 1.2 cells. $\mathrm{mm}^{2}, P=0.0054$ by Mann Whitney U test; Figure 7A). The number of p-JNK positive cells that co-localized with Fluoro-Jade B positive signals in the ischemic retinas only after 6 hours of reperfusion was significantly increased compared to that of the control retinas $\left(1.58 \pm 1.5\right.$ versus $0.0 \pm 0.0$ cell $/ \mathrm{mm}^{2}$, $P=0.0011$ by Mann Whitney U test; Figure 7A). In ischemic retinas, less than one-half of the $\mathrm{p}-\mathrm{JNK}$ positive cells expressed Fluoro-Jade B in the GCL (Figure 7B). Very few cells that expressed $\mathrm{p}-\mathrm{JNK}$ in the INL of ischemic retinas were costained with Fluoro-Jade B (Figure 7C and D). The Fluoro-Jade B positive cells had large soma (Figure 7E).

\section{Discussion}

Fluoro-Jade B is a well-established marker of degenerating neurons in the brain and retina (Schmued et al 1997; Fernandes et al 2004; Chang et al 2006, 2007; Oshitari et al 2008b). Fluoro-Jade B is an anionic derivative of fluorescein that interacts with intracellular polyamines and thereby stains degenerating neurons (Schmued and Hopkins 2000). FluoroJade $\mathrm{B}$ is a more specific marker for degenerating neurons than Fluoro-Jade because of the lower background staining, and the sharper demarcation of neurites. We used Fluoro-Jade $\mathrm{B}$ to identify degenerating neurons to determine the mechanisms of neuronal cell death in ischemia injured retinas.

The peak number of Fluoro-Jade B-positive cells in the GCL was found later than the peak number of TUNELpositive cells in the GCL of ischemic retinas (Kuroiwa et al 1998; Yokoyama et al 2001). This was probably because Fluoro-Jade B could not differentiate apoptosis from necrosis (Mundt-Petersen et al 2000; Ballok et al 2003). The number of Fluoro-Jade B-positive cells was relatively small after central retinal artery ligation models compared to excitotoxic reagents including kainic acid models (Chang et al 2007). The ischemic injury of our clipping model may be milder than the intravitreal injection of excitotoxic reagents model. 
A

$$
\left(\text { cells } / \mathrm{mm}^{2}\right)
$$

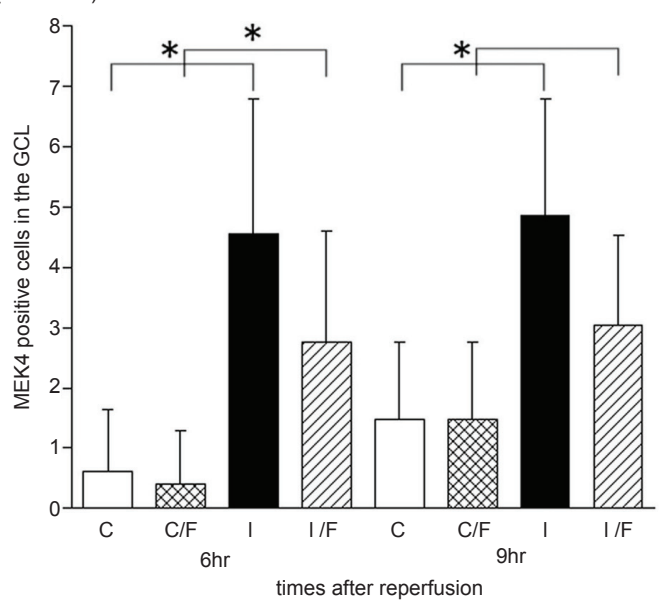

C

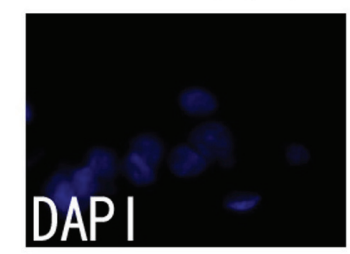

Ischemia
B

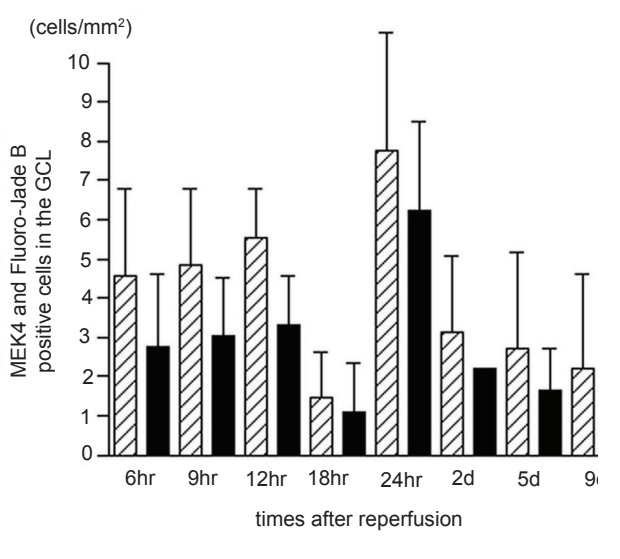

Control
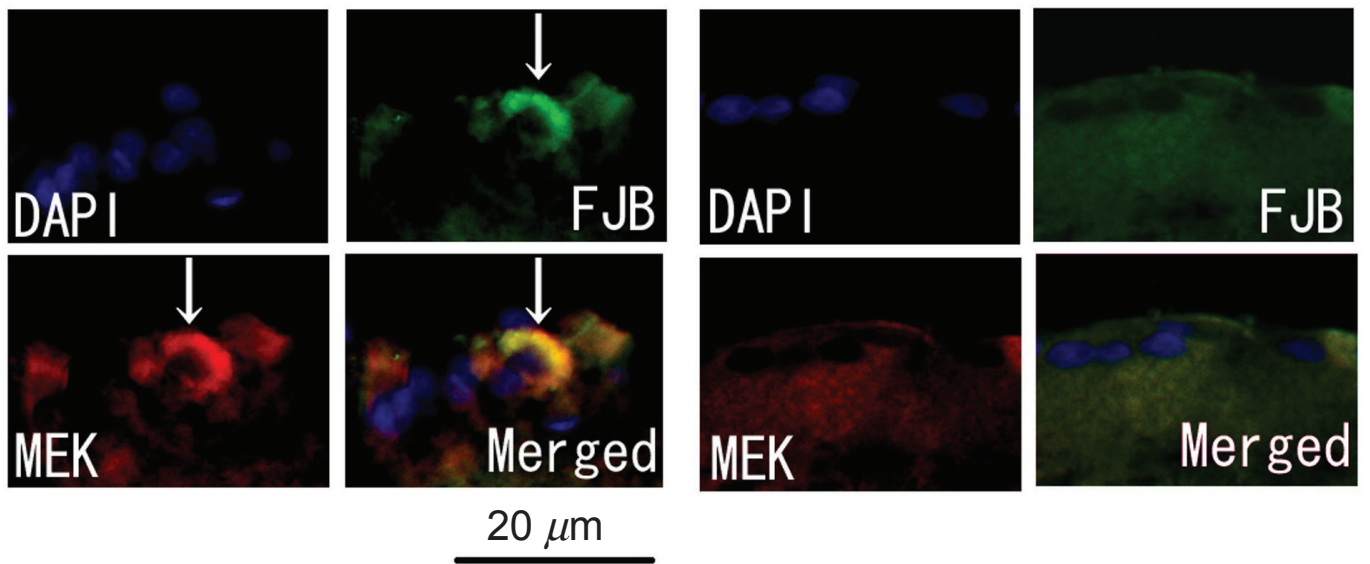

Figure 6 P-SEKI/MEK4 expression co-localized with Fluoro-Jade B-positive cells in the GCL and the time course of the number of p-SEKI/MEK4 positive cells in the GCL. The numbers of P-SEKI/MEK4 positive cells in the GCL in the ischemic retinas were significantly increased compared to those of the control retinas only after 6 hours and 9 hours of reperfusion (A).The number of P-SEKI/MEK4 positive and Fluoro-Jade B positive cells in the GCL in the ischemic retinas was significantly increased compared to that of the control only after 6 hours of reperfusion (A). In ischemic retinas, more than one-half of the cells that were p-SEKI/MEK4-positive were also Fluoro-Jade B-positive in the GCL (B).The sections were obtained from ischemic retinas 6 hours after reperfusion (C) (multi panel). The expression of p-SEKI/MEK4 is co-localized with Fluoro-Jade B-positive cells in the GCL (arrows) (C). (A); White bars showed the number of p-SEKI/MEK4 positive cells in the control retinas and checked bars showed the number of $\mathrm{P}$-SEKI/MEK4 positive and Fluoro-Jade B positive cells in the control retinas. Black bars showed the number of p-SEKI/MEK4 positive cells in the ischemic retinas and stripe bars showed $\mathrm{p}$-SEKI/MEK4 positive and Fluoro-Jade $\mathrm{B}$ positive cells in the ischemic retinas.

Abbreviations: C, control; I, Ischemia; C/F, Control (P-SEKI/MEK4) co-existed with Fluoro-Jade B; I/F, Ischemia (P-SEKI/MEK4) co-existed with Fluoro-Jade B; hr, hours; d, days. Notes: ${ }^{*} p<0.05$ by Mann Whitney $U$ test. (B); Stripe bars showed the number of $p$-SEKI/MEK4 positive cells and black bars showed the number of $p$-SEKI/MEK4 positive cells co-existed with Fluoro-Jade B-positive cells in the GCL of ischemic retinas.

In our model, total $50 \%$ of the RGCs in the ischemic retinas were degenerated after 7 days of reperfusion (Yokoyama et al 2001). On the other hand, in a NMDA and glycine injection model, over $80 \%$ of RGCs were dead (Manabe and Lipton 2003). Thus, the number of degenerating neurons may be fewer than we expected in the central retinal artery ligation models. However to identify the factors involved in neuronal degeneration, Fluoro-Jade B is a useful method because degenerating neurons are easily detected and the immunohistochemical staining with cell death-related factors is helpful in identifying which factors are associated with the neuronal death (Oshitari et al 2008b).
The ER plays a role in cellular homeostasis by inducing the unfolding protein response (UPR). At least three types of ER transmembrane proteins including IRE1 (protein kinase and site-specific endoribonuclease), PKR-like endoplasmic reticulum kinase, and activating transcriptional factor 6 are involved in the UPR. Urano and colleagues (2000) suggested that IRE1 activates JNK through the recruitment of tumor necrosis factor receptor-associated factor 2 (TRAF2), which is similar to the tumor necrosis factor- $\alpha$ (TNF- $\alpha$ )-induced apoptotic pathway. Our data suggest that the increased expression of IRE1 $\alpha$ was induced as a stress response and was associated with the pathogenesis 
A

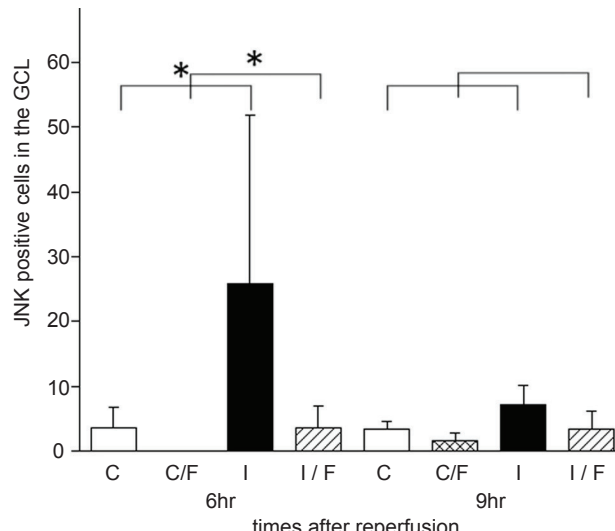

B
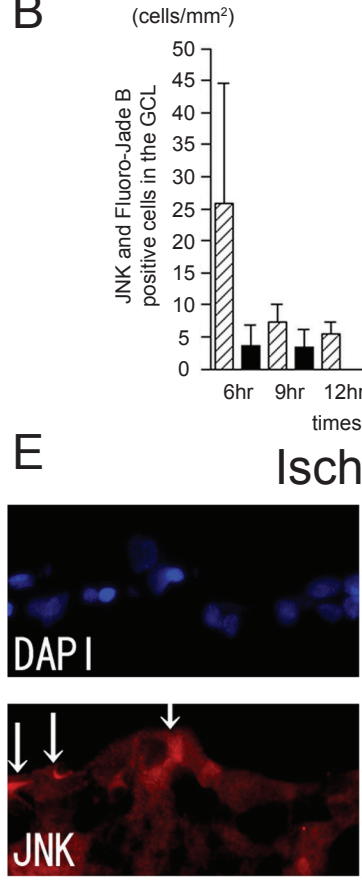

schemia
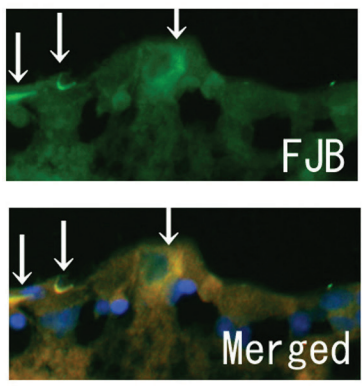

$20 \mu \mathrm{m}$
C
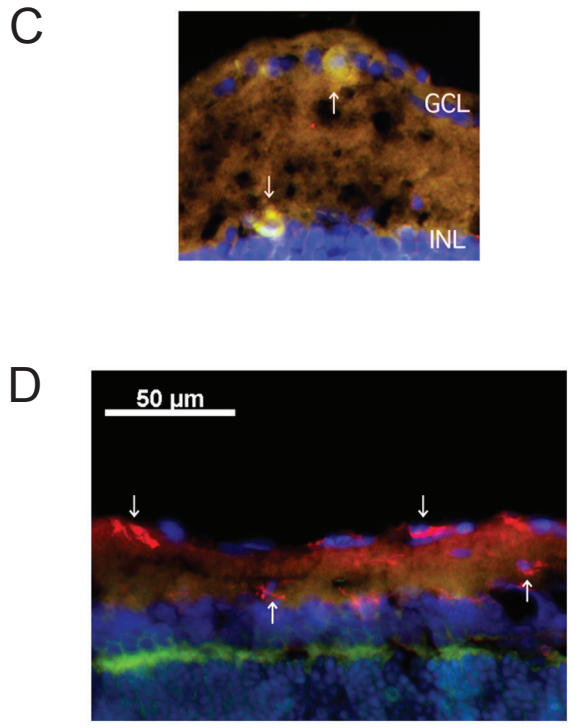

Control
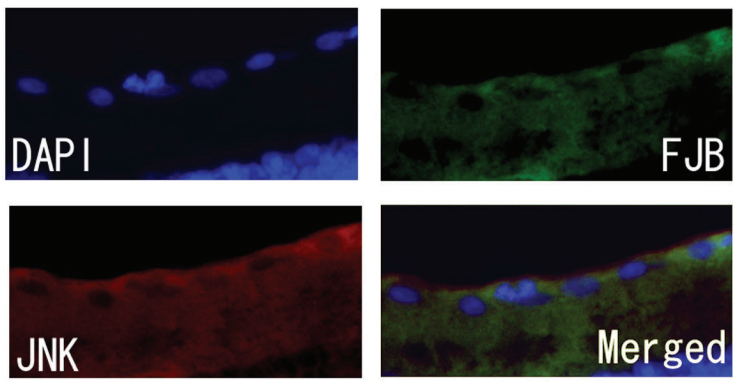

Figure 7 Expression of p-JNK in ischemic retinas and the time course of the number of p-JNK positive cells in the GCL.The numbers of p-JNK positive cells and p-JNK/FluoroJade B positive cells in the ischemic retinas were significantly increased compared to those of the control retinas only after 6 hours of reperfusion (A). Less than one-half of the p-JNK-positive cells were also Fluoro-Jade B-positive (B). The sections were obtained from ischemic retinas 6 hours after reperfusion (C-E). The expression of p-JNK co-existed with Fluoro-Jade B-positive cells in the GCL and the INL (arrows) (C) (merged) and (E) (multi panel). However, many type of cells with no Fluoro-Jade B positive signals were p-JNK-positive (D) (merged). Thus, JNK may play different roles in ischemic retinas as well as neuronal degeneration. (A); White bars showed the number of $\mathrm{P}$-JNK positive cells in the control retinas and checked bars showed the number of P-JNK positive and Fluoro-Jade B positive cells in the control retinas. Black bars showed the number of $\mathrm{p}$-JNK positive cells in the ischemic retinas and stripe bars showed $\mathrm{p}-\mathrm{JNK}$ positive and Fluoro-Jade $\mathrm{B}$ positive cells in the ischemic retinas.

Abbreviations: C, control; I, Ischemia; C/F, Control (P-JNK) co-existed with Fluoro-Jade B; I/F, Ischemia (P-JNK) co-existed with Fluoro-Jade B; hr, hours; d, days.

Notes: $* P<0.05$ by Mann Whitney $U$ test. (B); Stripe bars showed the number of p-JNK positive cells and black bars showed the number of $p$-JNK positive cells co-localized with Fluoro-Jade B-positive cells in the GCL of ischemic retinas.

of ischemic retinas. However, most of IRE1 $\alpha$ expression was increased in nondegenerative neurons. Thus, we could not determine whether the increased expression of IRE $1 \alpha$ was related to neuronal cell death, survival or recovery in ischemic retinas. A recent study showed that attenuation of the IRE1 $\alpha$ signal by persistent ER stress is related to cell death (Lin et al 2007). On the other hand, in the experimental stroke model, IRE1 expression is continuously increased and related to spontaneous recovery after the ischemic stress
(Rissanen et al 2006). Although our data are similar to those of the Rissanen and colleagues' study, further functional study is needed to examine the role of IRE1 $\alpha$ expression in damaged retinal neurons under ischemic stress.

Our data suggested that the increased expression of ASK1 was associated with neuronal death mainly in the GCL of ischemic retinas. These results are consistent with an earlier report (Harada et al 2006). In addition, our data showed that SEK1/MEK4 was phosphorylated and was related to 
neuronal cell death in the GCL of ischemic retinas. Both ASK1 and SEK1/MEK4 expression in the GCL was significantly increased only at the initial phase after reperfusion. It is well known that under ischemic stress, the concentration of intracellular $\mathrm{Ca}^{2+}$ is increased, which in turn leads to disturbances in the $\mathrm{Ca}^{2+}$ homeostasis in the ER (Verkhratsky and Toescu 2003). An upset of this homeostasis causes ER stress-induced neuronal death. An increase of intracellular $\mathrm{Ca}^{2+}$ concentration can activate SEK1/MEK4 because SEK1/MEK4 is activated under transiently high concentrations of $\mathrm{Ca}^{2+}$ in B cells (Dolmetsch et al 1997). Although the increased expression of ASK 1 and SEK1/MEK4 in degenerating neurons may have a causal relationship, further study is needed to examine the causal relationship between ER stress and ASK1-SEK1/MEK4 expression in degenerating neurons of ischemic retinas.

Our data suggested that JNK was phosphorylated and partly associated with neuronal cell death in the GCL of ischemic retinas. JNK expression as well as ASK1 and SEK1/ MEK4 expression in the GCL was significantly increased only at the initial phase after reperfusion. Roth and colleagues (2003) reported that JNK expression was significantly increased within 6 hours after ischemia-reperfusion. Our data on JNK expression at the initial phase after reperfusion is consistent with their report.

Similar to a past report (Roth et al 2003), our data showed that JNK expression was detected in many types of cells that were not Fluoro-Jade B positive in ischemic retinas. These results indicated that JNK may be, at least in part, associated with other molecular events than neuronal death in various types of retinal cells of ischemic retinas.

TNF- $\alpha$ can recruit TRAF2 and activate the ASK1-JNK pathways (Ichijo et al 1997). Tezel and Wax (2000) suggest that TNF- $\alpha$ is released from glial cells and induce RGC apoptosis under ischemic stress. On the other hand, Diem and colleagues (2001) stated that TNF- $\alpha$ rescued damaged RGCs by reducing the potassium currents and Akt phosphorylation. Thus, the role of TNF- $\alpha$ in RGC death is complex. TNF- $\alpha$ may be related to ASK1-JNK pathway that is activated in ischemic retinas.

Most Fluoro-Jade B positive cells had large soma which is characteristic of RGCs. The ischemic stress may be the cause of the onset and the progression of the pathogenesis including RGC death in diabetic retinopathy and glaucoma (Oshitari and Roy 2007). Thus our data may provide some clue on common cell death mechanisms of damaged RGCs involved in the pathogenesis of diabetic retinopathy and glaucoma. Although the evidence is circumstantial, our date showed an association of IRE1 $\alpha$, ASK1, SEK1/MEK4, and JNK expression with neuronal cell death of ischemic retinas. Further functional studies are needed to examine the relation between ER stress and the signal transduction proteins expression including ASK1, SEK1/MEK4, and JNK in ischemic retinas.

\section{Conclusions}

Our results suggest that an increased expression of ER stress-related factors IRE1 $\alpha$, ASK1, SEK1/MEK4, and JNK is associated with the neuronal abnormalities of ischemiareperfusion injury in rat retinas. Further functional studies are needed to determine whether the ER stress is associated with the increased expression of these stress-related signal transduction proteins in neuronal cell death in ischemic retinas.

\section{Acknowledgments}

This study was supported by the grant from The Eye Research Foundation for the Aged (T.O) and the Grant-in-Aid for Scientific Research from Ministry of Education, Science, Sports and Culture (YM and SY). We thank Prof. Duco Hamasaki for editing this manuscript.

\section{References}

Awai M, Koga T, Inomata Y, et al. 2006. NMDA-induced retinal injury is mediated by an endoplasmic reticulum stress-related protein, $\mathrm{CHOP} /$ GADD153. J Neurochem, 96:43-52.

Ballok DA, Millward JM, Sakic B. 2003. Neurodegeneration in autoimmune MRL-lpr mice as revealed by Fluoro Jade B staining. Brain Res, 964:200-10.

Chang ML, Wu CH, Chien HF, et al. 2006. Microglia/macrophages responses to kainate-induced injury in the rat retina. Neurosci Res, 54:202-12.

Chang ML, Wu CH, Jiang-Shieh YF, et al. 2007. Reactive changes of retinal astrocytes and Muller glial cells in kainate-induced neuroexcitotoxicity. J Anat, 210:54-65.

Diem R, Meyer R, Weishaupt JH, et al. 2001. Reduction of potassium currents and phosphatidylinositol 3-kinase-dependent AKT phosphorylation by tumor necrosis factor-(alpha) rescues axotomized retinal ganglion cells from retrograde cell death in vivo. J Neurosci, 21:2058-66.

Dolmetsch RE, Lewis RS, Goodnow CC, et al. 1997. Differential activation of transcription factors induced by $\mathrm{Ca}^{2+}$ response amplitude and duration. Nature, 386:855-8.

Fernandes AM, Maurer-Morelli CV, Campos CB, et al. 2004. Fluoro-Jade, but not Fluoro-Jade B, stains non-degenerating cells in brain and retina of embryonic and neonatal rats. Brain Res, 1029:24-33.

Harada C, Nakamura K, Namekata K, et al. 2006. Role of apoptosis signalregulating kinase 1 in stress-induced neural cell apoptosis in vivo. $\mathrm{Am}$ J Pathol, 168:261-9.

Hayashi T, Saito A, Okuno S, et al. 2005. Damage to the endoplasmic reticulum and activation of apoptotic machinery by oxidative stress in ischemic neurons. J Cereb Blood Flow Metab, 25:41-53.

Imai Y, Takahashi R. 2004. How do Parkin mutations result in neurodegeneration? Curr Opin Neurobiol, 14:384-9.

Ichijo H, Nishida E, Irie K, et al. 1997. Induction of apoptosis by ASK1, a mammalian MAPKKK that activates SAPK/JNK and p38 signaling pathways. Science, 275:90-4. 
Kuroiwa S, Katai N, Shibuki H, et al. 1998. Expression of cell cycle-related genes in dying cells in retinal ischemic injury. Invest Ophthalmol Vis Sci, 39:610-17.

Larner SF, Hayes RL, McKinsey DM, et al. 2004. Increased expression and processing of caspase-12 after traumatic brain injury in rats. J Neurochem, 88:78-90.

Levin LA, Louhab A. 1996. Apoptosis of retinal ganglion cells in anterior ischemic optic neuropathy. Arch Ophthalmol, 114:488-91.

Lindholm D, Wootz H, Korhonen L. 2006. ER stress and neurodegenerative diseases. Cell Death Differ, 13:385-92.

Manabe S, Lipton SA. 2003. Divergent NMDA signals leading to proapoptotic and antiapoptotic pathways in the rat retina. Invest Ophthalmol Vis Sci, 44:385-92.

Mundt-Petersen U, Petersen A, Emgard M, et al. 2000. Caspase inhibition increases embryonic striatal graft survival. Exp Neurol, 164:112-20.

Nishitoh H, Matsuzawa A, Tobiume K, et al. 2002. ASK1 is essential for endoplasmic reticulum stress-induced neuronal cell death triggered by expanded polyglutamine repeats. Genes Dev, 16:1345-55.

Oshitari T, Hata N, Yamamoto S. 2008a. Endoplasmic reticulum stress and diabetic retinopathy. Vasc Health Risk Manag, 4:115-22.

Oshitari T, Roy S. 2007. Common therapeutic strategies for diabetic retinopathy and glaucoma. Cur Drug Ther, 2:224-32.

Oshitari T, Yamamoto S, Hata N, et al. 2008b. Mitochondria- and caspasedependent cell death pathway involved in neuronal degeneration in diabetic retinopathy. Br J Ophthalmol, 92:552-6.

Rao RV, Ellerby HM, Bredesen DE. 2004. Coupling endoplasmic reticulum stress to the cell death program. Cell Death Differ, 11:372-80.

Roth S, Shaikh AR, Hennelly MM, et al. 2003. Mitogen-activated protein kinases and retinal ischemia. Invest Ophthalmol Vis Sci, 44:5385-95.

Shimazawa M, Inokuchi Y, Ito Y, et al. 2007. Involvement of ER stress in retinal cell death. Mol Vis, 13:578-87.

Schmued LC, Albertson C, Slikker W Jr. 1997. Fluoro-Jade: a novel fluorochrome for the sensitive and reliable histochemical localization of neuronal degeneration. Brain Res, 751:37-46.
Schmued LC, Hopkins KJ. 2000. Fluoro-Jade B: a high affinity fluorescent marker for the localization of neuronal degeneration. Brain Res, 874:123-30.

Tajiri S, Oyadomari S, Yano S, et al. 2004. Ischemia-induced neuronal cell death is mediated by the endoplasmic reticulum stress pathway involving CHOP. Cell Death Differ, 11:403-15.

Towbin H, Staehelin T, Gordon J. 1979. Electrophoretic transfer of proteins from polyacrylamide gels to nitrocellulose sheets: procedure and some applications. Proc Nalt Acad Sci US A, 76:4350-4.

Tezel G, Wax MB. 2000. Increased production of tumor necrosis factoralpha by glial cells exposed to simulated ischemia or elevated hydrostatic pressure induces apoptosis in cocultured retinal ganglion cells. J Neurosci, 20:8693-700.

Tobisawa S, Hozumi Y, Arawaka S, et al. 2003. Mutant SOD1 linked to familial amyotrophic lateral sclerosis, but not wild-type SOD1, induces ER stress in COS7 cells and transgenic mice. Biochem Biophys Res Commun, 303:496-503.

Urano F, Bertolotti A, Ron D. 2000. IRE1 and efferent signaling from the endoplasmic reticulum. J Cell Sci, 113(Pt 21):3697-702.

Verkhratsky A, Toescu EC. 2003. Endoplasmic reticulum $\mathrm{Ca}\left(2^{+}\right)$ homeostasis and neuronal death. J Cell Mol Med, 7:351-61.

Wootz H, Hansson I, Korhonen L, et al. 2004. Caspase-12 cleavage and increased oxidative stress during motoneuron degeneration in transgenic mouse model of ALS. Biochem Biophys Res Commun, 322:281-6.

Yokoyama A, Oshitari T, Negishi H, et al. 2001. Protection of retinal ganglion cells from ischemia-reperfusion injury by electrically applied Hsp27. Invest Ophthalmol Vis Sci, 42:3283-6.

Zhang Y, Cho CH, Atchaneeyasakul LO, et al. 2005. Activation of the mitochondrial apoptotic pathway in a rat model of central retinal artery occlusion. Invest Ophthalmol Vis Sci, 46:2133-9. 